\title{
Automatic visual bias of perceived auditory location
}

\author{
PAUL BERTELSON \\ Université Libre de Bruxelles, Brussels, Belgium \\ and Tilburg University, Tilburg, The Netherlands \\ and \\ GISA ASCHERSLEBEN \\ Max Planck Institute for Psychological Research, Munich, Germany
}

\begin{abstract}
Studies of reactions to audiovisual spatial conflict (alias "ventriloquism") are generally presented as informing on the processes of intermodal coordination. However, most of the literature has failed to isolate genuine perceptual effects from voluntary postperceptual adjustments. A new approach, based on psychophysical staircases, is applied to the case of the immediate visual bias of auditory localization. Subjects have to judge the apparent origin of stereophonically controlled sound bursts as left or right of a median reference line. Successive trials belong to one of two staircases, starting respectively at extreme left and right locations, and are moved progressively toward the median on the basis of the subjects' responses. Response reversals occur for locations farther away from center when a central lamp is flashed in synchrony with the bursts than without flashes (Experiment 1), revealing an attraction of the sounds toward the flashes. The effect cannot originate in voluntary postperceptual decision, since the occurrence of response reversal implies that the subject is uncertain concerning the direction of the target sound. The attraction is contingent on sound-flash synchronization, for early response reversals did no longer occur when the inputs from the two modalities were desynchronized (Experiment 2). Taken together, the results show that the visual bias of auditory localization observed repeatedly in less controlled conditions is due partly at least to an automatic attraction of the apparent location of sound by spatially discordant but temporally correlated visual inputs.
\end{abstract}

When subjects are asked to indicate, by pointing or some verbal report, the location of an auditory event that is accompanied by the presentation of synchronous but spatially discordant visual data, their response is typically dispiaced in the direction of the latter (Bermant \& Welch, 1976; Bertelson \& Radeau, 1981, 1987; Bertelson, Vroomen, Wiegeraad, \& de Gelder, 1994; Klemm, 1909; Radeau, 1992; Radeau \& Bertelson, 1987; Thomas, 1941; Warren, Welch, \& McCarthy, 1981). That visual bias of

The present work was presented at the XXVIth International Congress of Psychology, Montreal, 1996 (Bertelson, 1998), at the 1996 annual meeting of the Psychonomic Society in Chicago (Bertelson \& Aschersleben, 1996), at the 50th Anniversary Meeting of the Belgian Psychological Society, and at the symposium on Varieties of Implicit Processing in Tilburg (The Netherlands), 1997. The contribution of the first author to the project was supported by the Belgian Fund for Collective Fundamental Research (FRFC), by the Ministry of Scientific Research of the Belgian French-Speaking Community (Concerted Research Actions 91/96-148 and 96/01-2037), and by Visiting Scientist Fellowships from the Max Planck Society. The computer programs were developed by Frank Miedreich, and Renate Tschakert helped with testing the subjects. Thanks are due Jim Cutting, Dom Massaro, Bob Welch, and an anonymous reviewer for their constructive examination of the manuscript. The work benefited also from discussions with Béatrice de Gelder, Jon Driver, Richard Held, Jacques Paillard, Wolfgang Prinz, and Jean Vroomen. Correspondence should be addressed to P. Bertelson, Laboratoire de Psychologie Expérimentale, U.L.B., $50 \mathrm{Av}$. F. D. Roosevelt, B-1050 Bruxelles, Belgium (e-mail: pbrtlsn@ulb.ac.be). auditory location ${ }^{1}$ is one of a set of phenomena observed in audiovisual spatial conflict situations which, besides immediate bias, involve nondetection of the spatial discrepancy (Bertelson \& Radeau, 1981; Choe, Welch, Gilford, \& Juola, 1975) and the occurrence of adaptive aftereffects (Canon, 1970; Radeau \& Bertelson, 1974, 1977). These phenomena have come to be collectively designated by the term "ventriloquism," since one of their most spectacular manifestations is the illusion created by performing ventriloquists that the speech that they produce without visible lip movements comes from the mouth of a simultaneously moved puppet.

One main reason to be interested in these various reactions to intermodal conflict is that they may help us understand the mechanisms through which a coherent representation of extrapersonal space is established and maintained. Through the fast growing body of data on crossmodal convergence at the neuronal level (e.g., Graziano \& Gross, 1996; Stein \& Meredith, 1993) they might be related to basic physiological processes. However, data from localization performance are relevant to the general problem of intermodal coordination only if they originate in genuine perceptual processes and not simply in voluntary response strategies adopted to satisfy the demands of the particular experimental situation. Isolating the contributions of automatic perceptual processes from those of postperceptual decisions is of course 
a problem for the interpretation of all kinds of evidence based on the reports of human subjects (for a similar example involving the use of the "familiar size" cue for visual distance estimation, see Predebon \& Woolley, 1994). In the case of ventriloquism, it so happens that most studies in the literature could have been contaminated to some degree by postperceptual adjustments.

Studies of immediate reactions are especially susceptible to voluntary influences to the extent that the instructions draw the subject's attention to the possibility of a discrepancy. For instance, the visual bias of auditory location has typically been studied by presenting target auditory stimuli in one location and visual distractors in a different location, and asking the subject to point to the location of the auditory stimuli and to ignore the visual distractors (e.g., Bermant \& Welch, 1976). The subject is then left free to speculate about the reasons why the latter are presented at all, and to adjust her/his response strategy in consequence.

An indirect argument for the existence of a genuine perceptual component in ventriloquism has sometimes been derived from the fact that exposure to conflicting bimodal data results in adaptive aftereffects. The notion, which has been traditional in work on intermodal conflict (see, e.g., Welch, 1978, pp. 8-9), is that aftereffects are measured in a straightforward unimodal localization task, in which there is less reason to engage in voluntary correction than when one is reacting in the presence of the conflicting data. The possibility cannot completely be excluded, however, that conscious detection of the experimental discrepancy during the exposure phase influences the setting of response criteria during the posttests. On the other hand, even if the argument from aftereffects was fully convincing, it would still be necessary, for the sake of coherence, to demonstrate the existence of a perceptual component in the immediate effects themselves.

The first direct contribution to that issue was made in a study by Choe et al. (1975), who found that synchronization of auditory and visual inputs increased the frequency of "same" judgments by subjects who had been asked to judge the origin of sound bursts and light flashes delivered in the same or in different places. Application of detection theory to the results showed that synchronization affected the decision criterion $\beta$ and not $d^{\prime}$. The authors concluded that ventriloquism originated in a response bias, not in shifts of the perceived locations of the inputs.

In a comment on the Choe et al. (1975) paper, however, Bertelson and Radeau (1976) argued that the particular perceptual effect of synchronization that the detection analysis had discarded was from the start implausible, and that a more likely one was compatible with the results. ${ }^{2}$ They proposed that an effect of synchronization on the registered locations of the inputs was still a valid possibility. On the other hand, they insisted that crossmodal effects on localization performance could also originate at postperceptual processing stages, like response biases or even deliberate judgments. These possibilities have arisen especially in studies involving or simulating situations known to produce correlated inputs to the ear and eye, such as speech and the face of the speaker (Bertelson et al., 1994; Radeau \& Bertelson, 1977, Experiment 2; Witkin, Wapner, \& Leventhal, 1952), or the sound and the sight of a familiar noisy event (Canon, 1970; Jackson, 1953; Radeau \& Bertelson, 1977, Experiment 1). For instance, Pick, Warren, and Hay (1969) reported that the location in which subjects localized sound bursts was biased in the direction of a loudspeaker seen at some distance to the side of the auditory source. The occurrence of bias in the absence of any visual change to correlate with the bursts was obviously contingent on the subjects' knowledge of the function of loudspeakers. Thus, the effect presumably originated in some postperceptual adjustments. ${ }^{3}$

An alternative to Choe et al.'s (1975) attempt at localizing the origin of ventriloquism through detection analysis is to improve the tasks. Our earlier discussion of the unimodal localization task led to the conclusion that its main inconvenience was transparency: When the subject is aware both of the spatial separation and of the degree of synchronization of the data, all the conditions for the operation of postperceptual adjustments are met. Thus the principal requirement for demonstrating the existence of a perceptual contribution to crossmodal bias would be to observe the effect in the absence of conscious detection of the discrepancy.

Results that went some way in that direction were obtained by Bertelson and Radeau (1981, Experiment 2) in an experiment in which sound bursts and light flashes were presented simultaneously at varying separations, and on each trial the subjects both pointed to the location of the sounds and judged whether the sounds and the flashes had come from the same place or from different places. Thus, visual bias could be examined separately for trials on which the spatial discrepancy was detected and trials on which it was not. However, detection of the discrepancy is presumably influenced by trial-to-trial variations in the perceived location of both sounds and flashes, and the measured biases had to be corrected ${ }^{4}$ for that influence. The final outcome was that the corrected visual biases of sound localization were significant for both detection and no-detection trials. The finding of bias in the absence of detection of the discrepancy suggested a negative answer to the question of the role of awareness of the discrepancy in the generation of crossmodal bias. However, the conclusions one could draw from that particular experiment depended on the validity of the correction procedure, and for that reason needed confirmation by a more direct method.

In the present study, we introduce a straightforward experimental approach to the study of bias which we think meets the nontransparency condition. Trains of sound pulses are presented in varying azimuthal locations, and for each presentation the subject gives a dichotomic leftright judgment. The location of the target is changed as a function of those judgments, following the principle of the psychophysical staircase (Cornsweet, 1962; Levitt, 
1971). After a "left" judgment, the next target on the same staircase is moved one step to the right, and vice versa. Two staircases are run in random alternation, starting from two extreme locations respectively to the left and to the right. The two staircases necessarily converge progressively toward a median location. Initially, the correct response is provided repeatedly on each staircase, so that, except for occasional mistakes, the progression toward the center is monotonic. Then, at some point, response reversals (i.e., responses different from the preceding one) begin to occur. From this point on, the subject is apparently uncertain regarding the accuracy of her/his judgment, and the nontransparency condition is met.

To examine the possibility of visual bias, light flashes are produced in a central location in synchrony with the sound pulses. Attraction of the apparent location of the sounds by the flashes should manifest itself by the occurrence of response reversals for locations farther away from center with synchronized flashes than without them, or than with other less effective visual inputs.

In a pilot experiment (described briefly in Bertelson, 1998), the condition with synchronized flashes was compared with a control condition in which the central lamp stayed on continuously. As had been predicted, the convergence of the two staircases was slowed in the condition with synchronized flashes in comparison with the one with the steady light. The result could not be unambiguously attributed to the different stimulus arrangements, however, because the two conditions were presented in the same order-steady light then flashing light- - to all subjects.

\section{EXPERIMENT 1}

The purpose of this experiment was to determine whether presenting, in a central location, light flashes synchronized with the sounds would result in the occurrence of response reversals at earlier stages on the staircases than is the case with no synchronized flashes. In the control condition, the central lamp was invisible and the task was to judge the location of the sounds relative to a central meridian line. The order of presentation of the conditions was counterbalanced, avoiding the confounding of the effect of conditions with that of practice which compromised the interpretation of the results from the pilot experiment.

\section{Method}

Subjects. Ten paid volunteers from the subjects panel of the Max Planck Institute ( 7 female and 3 male; mean age, 28 years) participated in two sessions each

Experimental situation. Each subject sat, with her/his head in an adjustable forehead and chin rest, in front of a vertical dark curtain with a white vertical string in median position. An LED lamp was positioned at about eye level behind the cloth. It was invisible when off, but shined through the curtain when lit. Two loudspeakers were positioned behind the curtain at $50 \mathrm{~cm}$ on either side of the middle and at the same level as the LED. On each trial, three $15-\mathrm{msec}$ pulses of $2000-\mathrm{Hz}$ sinewave sound separated by $800-\mathrm{msec}$ intervals were presented. The apparent azimuthal origin of the sounds was controlled by manipulating the phase difference between emissions on the two loudspeakers. The task was to indicate, by pressing one of two keys on a standard keyboard, whether the sound came from the left or from the right of the vertical meridian. A Hewlett-Packard Vectra QS/20 personal computer controlled the presentation of the stimuli via a $\mathrm{D} / \mathrm{A}$ converter and a Sony TA-F170 amplifier and also recorded the responses.

Procedure. Testing was organized into explorations. For each exploration, successive trials were selected from two randomly intermingled staircases, one starting with the sounds at the extreme left position and the other at the extreme right. When the "left" key was pressed, the following sound on the same staircase was moved one step to the right, and vice versa after a "right" key press. For each trial, the staircase controlling the location of the auditory target was chosen at random. At some point on each staircase, reversals, defined as responses different from the preceding one on the same staircase, began to occur. An exploration was stopped automatically as soon as 10 reversals had been recorded on each of the two staircases. Each staircase started with steps equal to 2 phase difference units, and shifted to one unit steps as soon as a threshold of 10 units from 0 was crossed. The phase difference unit was $40 \mu \mathrm{sec}$, approximately equivalent to an angular separation of $2.4^{\circ}$ of angle. ${ }^{5}$ Each trial started $2 \mathrm{sec}$ after the response to the preceding one. An exploration generally took 5-8 min.

Explorations were run under two conditions. In the SF (for "simultaneous flash") condition, one 15-msec flash in the LED lamp occurred simultaneously with each sound pulse. In the NF ("no flash") condition, the sounds occurred without any accompanying visual event. This condition was thus different from the control condition used in the pilot experiment, which involved the LED permanently on. The change of control condition was motivated by the notion that the steady light might by itself produce some limited degree of attraction.

For both conditions, subjects were instructed to keep looking at the central vertical line. In Condition SF, $10 \%$ of catch trials, on which the color of one of the three flashes changed from yellowish to reddish, were interspersed at random intervals. For those trials, subjects were instructed to press the space bar of the keyboard instead of one of the normal response keys. To allow normal continuation of the staircases, the sound location at which a catch trial occurred was presented again on the following trial on the same staircase.

Every session involved two blocks of three explorations, one block under Condition SF and the other under Condition NF. The order of administration of the conditions over the two sessions was balanced across subjects

\section{Results}

Two sample explorations by the same subject, one under each of the two conditions, are shown in Figure 1. In the exploration under Condition NF (left graph), the two staircases reach a point where they cross each other. On the contrary, for the exploration under Condition SF (right graph), the two staircases start reversing when they are still wide apart and, as a result, remain separate up to the point at which the criterion of 10 reversals on each staircase is reached. The difference between explorations under the two conditions was not always as extreme as in these two examples, which were chosen just to exemplify the approach. On the other hand, even under Condition SF, reversals occur only when the staircases have converged substantially toward some medial position. This result was typically obtained in most explorations. ${ }^{6}$

The analysis of the data was based on the locations at which the first 10 response reversals occurred on each staircase. To help illustrate the method, the dots corresponding to these 10 first reversals are filled on the stair- 

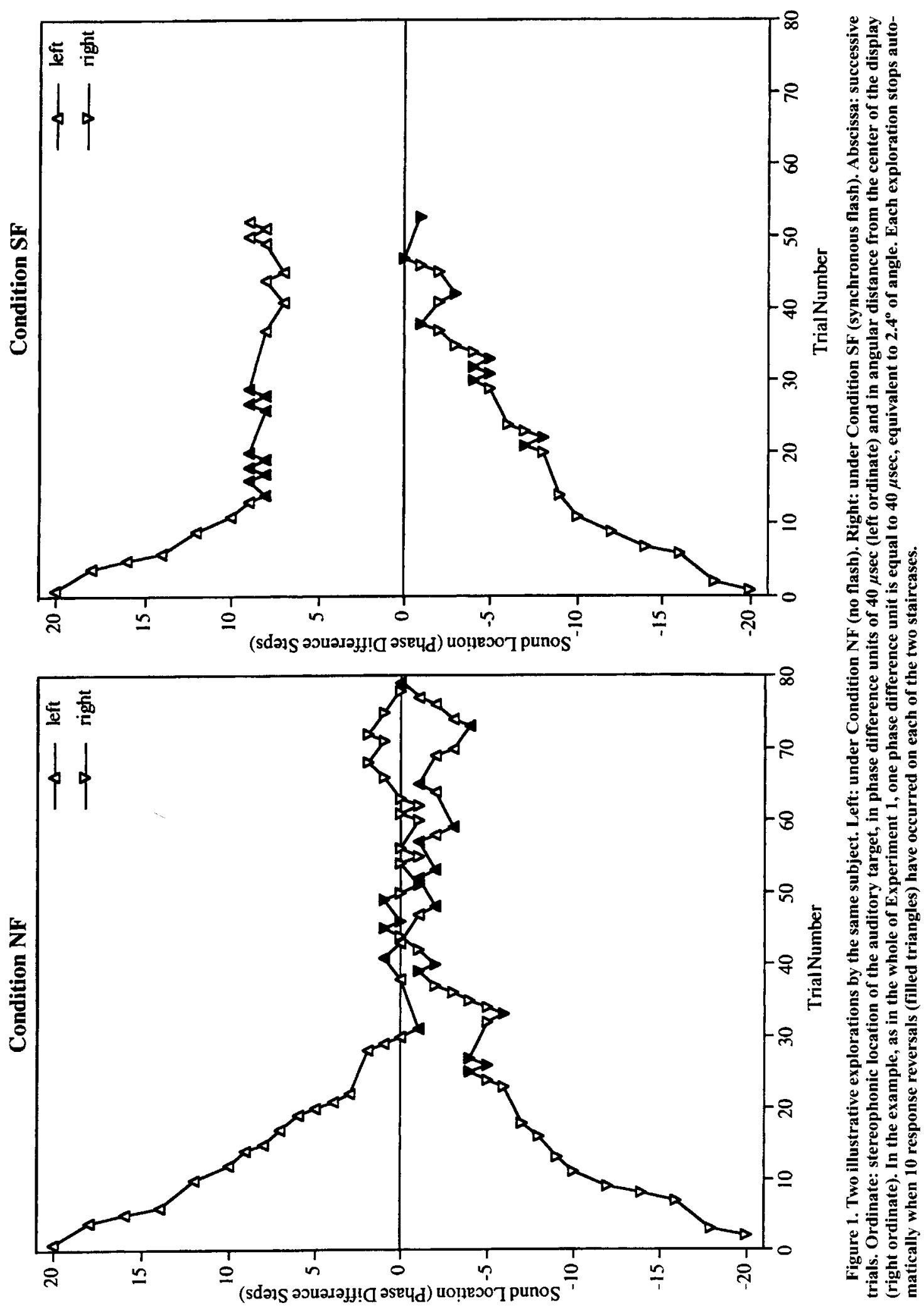


\section{Experiment 1}
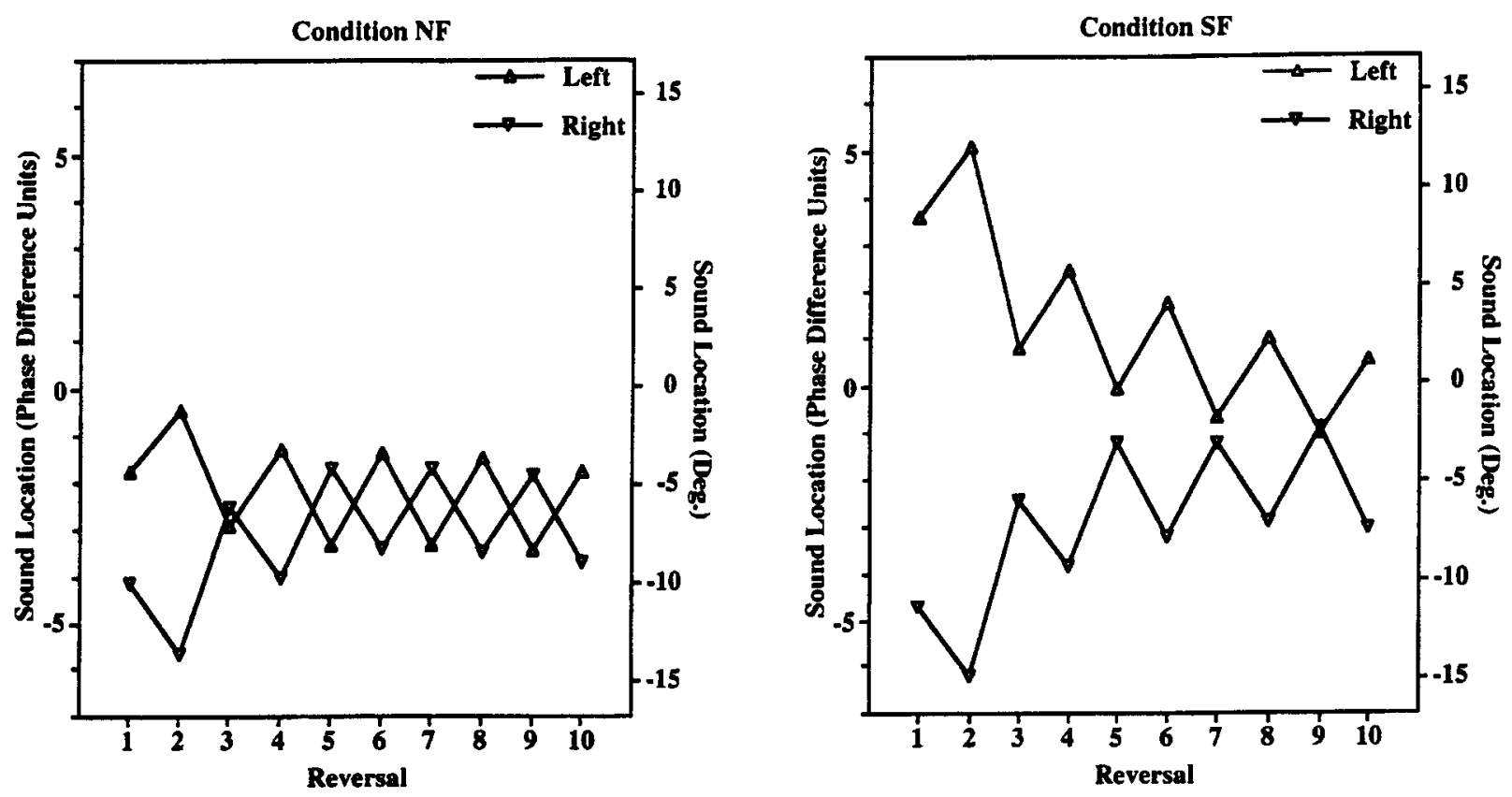

\section{Experiment 2}
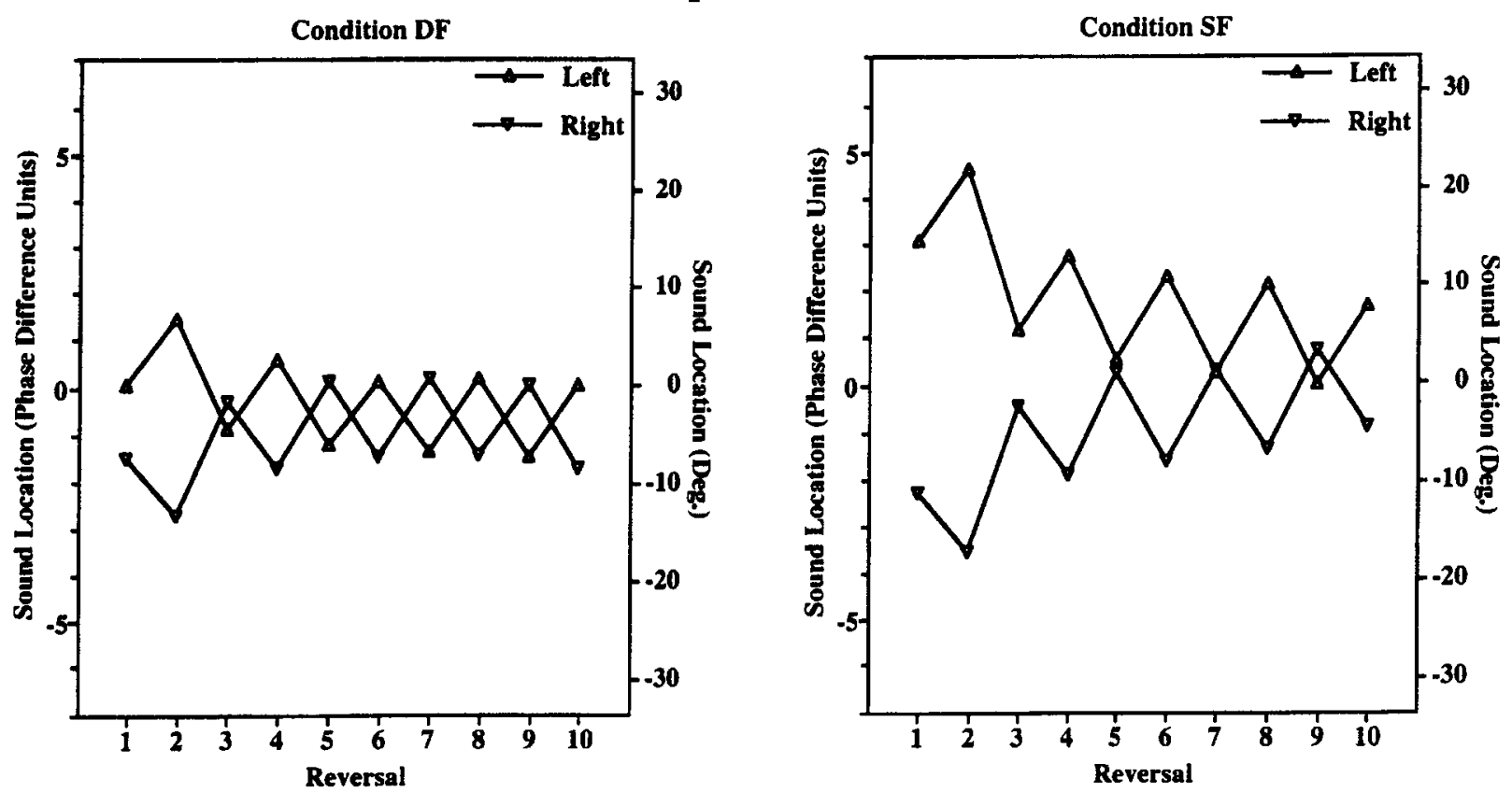

Figure 2. Mean location, in phase difference units (left ordinate) and in degrees of angle (right ordinate), of 10 first response reversals on each staircase. For reasons explained in the Method section of Experiment 2, the phase difference unit was larger ( $80 \mu$ sec, equivalent to $4.8^{\circ}$ of angle) in that experiment than in Experiment 1 (40 $\left.\mu \mathrm{sec}, 2.4^{\circ}\right)$. NF, no flash; SF, simultaneous flash; DF, desynchronized nash.

cases of Figure 1. Means across explorations and subjects of the locations of these 10 reversals are shown, separately for the two conditions, in Figure 2 (upper two graphs). The sawtooth appearance of the curves is an unavoidable consequence of the method: Successive reversals are nec- essarily separated by at least one phase difference step. It is nevertheless quite apparent that the staircases converge more slowly under Condition SF than under Condition NF. The mean distances between the locations (measured in phase difference units) of the 10 first reversals on the left 
and the right staircases respectively are 1.1 units for Condition NF and 4.3 units for Condition SF. By a Student paired values $t$ test, the effect of conditions is significant $[t(9)=2.27, p<.025$, one-tailed $]$. The application of a one-tailed test is justified by the existence of a clear prediction concerning the direction of the difference. In addition, the overall pattern of larger interstaircases distance in Condition SF than in Condition NF is shown by 9 out of the 10 subjects, which by a simple sign test is significant at $p=.001$ (one-tailed).

In Condition NF, the mean staircases appear to converge to a location somewhat to the right of the middle. This tendency, which was not replicated in other experiments in the series (e.g., Experiment 2), is probably just a manifestation of sampling variability.

\section{Discussion}

The apparent location of the target sounds was attracted toward the synchronous flashing light. The effect, first obtained in the pilot experiment by comparing the flashing light condition with a control condition in which the light was steadily on, was now replicated with a nolight condition as a control, and with adequate balancing of the order of administration of the conditions. The evidence for attraction was obtained again at times when occurrence of response reversals demonstrated the observers' uncertainty regarding the location of the target. No voluntary strategy can thus explain the effect.

So far, the results show that the flashing light attracts the sound automatically. The choice as candidate attractors of flashes synchronized with the sounds was dictated of course by the evidence in the literature that synchronization of the inputs is an important condition for the occurrence of the various manifestations of ventriloquism. Strong effects of synchronization have been demonstrated for perceptual fusion (Choe et al., 1975; Jack \& Thurlow, 1973; Radeau \& Bertelson, 1977, Experiment 3), for immediate bias (Klemm, 1909; Radeau \& Bertelson, 1987; Thomas, 1941; Warren et al., 1981), and for aftereffects (Radeau \& Bertelson, 1977, Experiment 2). However, in the comparisons carried out between the condition with flashes synchronized with the tones and those with a steady light (pilot experiment) or with no light (Experiment 1), the influence of synchronization was confounded with that of light interruption. Some data of Thomas (1941), later confirmed by Radeau and Bertelson (1987), have in fact suggested that interrupted light flashes can to some extent bias the localization of nonsynchronous sounds, such as, for instance, steady ones. Thus, it could not yet be claimed that the particular automatic component of bias that we were trying to isolate was contingent on synchronization. The importance of synchronization per se was examined in the next experiment.

\section{EXPERIMENT 2}

The aim of this new experiment was to determine whether the attraction of sounds by the flashing light thus far observed depended on the fact that the flashes were synchronized with the sounds. A condition with synchronized flashes similar to the one used in the previous experiments was compared with one in which the sounds and the flashes were separated by unpredictably variable time intervals.

\section{Method}

Subjects. Twelve paid volunteers ( 8 female, 4 male; mean age, 28 years) from the Max Planck panel participated in two sessions each.

Experimental situation. The trains of three tones separated by $800 \mathrm{msec}$ which were used in the preceding experiment did not leave enough space for creating effective desynchronization. In the present experiment, only two 15 -msec tones, separated by $1,600 \mathrm{msec}$, were presented on each trial. In Condition SF (for "simultaneous flash", as in Experiment 1), one 15-msec flash always occurred in exact synchrony with each tone. In Condition DF (for "desynchronized flash"), there was around each tone a double temporal window within which one flash occurred on each trial. These windows extended from 800 to $400 \mathrm{msec}$ before tone onset and from 400 to $800 \mathrm{msec}$ after tone offset. The temporal locations of the two flashes of each trial were chosen independently by the computer, following a rectangular distribution of locations within each double window. To control the placement of the stimuli over the present range of values, it was necessary to reduce the period of the time base by a factor of two. The result is that phase difference units increased, in comparison with those in Experiment 1 , from 40 to $80 \mu \mathrm{sec}$, equivalent to an angular distance of $4.8^{\circ}$. Catch trials, on which the color of one of the two flashes was changed and the subject had to press the space bar instead of one of the normal response keys, occurred on about $10 \%$ of the trials under both conditions.

Procedure. The procedure was basically the same as in Experiment 1 . Each exploration proceeded until 10 reversals had been recorded on each staircase, and analysis was based on the locations of the 10 first reversals per staircase. As in Experiment 1, each subject performed one block of three explorations under each condition on each of two sessions, and the order of conditions over the sessions was balanced across subjects.

\section{Results and Discussion}

The data were analyzed in the same way as in Experiment 1 . Mean tone locations at which the first 10 reversals occurred are shown in Figure 2, in the lower left frame for Condition DF and lower right frame for Condition SF. The distance between the two staircases is smaller under Condition DF than under Condition SF across the successive reversals (for DF, $x=1.6$ phase difference units; for SF: $x=5.9$ units). The difference between the two conditions is significant $[t(11)=2,23$, $p<.025$, one-tailed]. Nine out of the 12 subjects had larger interstaircases distances under Condition SF than under Condition DF, which by one-tailed sign-test is significant at $p=.019$.

Thus, a significant effect of input synchronization on bias has now been demonstrated through a comparison between conditions equated regarding the occurrence of light interruption. This result shows that the effect of synchronization demonstrated in earlier studies of visual bias originates in part at least in its perceptual component.

It would be interesting to know how the size of the bias changes with degree of desynchronization. The purpose of the present experiment was only to establish the relevance of the factor of synchronization. Hence, the synchronous condition was compared with an extreme desynchronized condition with temporal separations between visual and auditory inputs that were both of minimum duration and variable. The respective roles of size and variability of temporal separations will be examined in future experiments.

\section{GENERAL DISCUSSION}

In the introduction, we argued that although crossmodal interactions in localization performance hold the promise of important implications regarding the basic mechanisms of coordination between the spatial senses, the problem of separating properly perceptual contributions to these interactions from those of judgmental postperceptual processes 
has not received enough attention. In the specific case of the visual bias of auditory localization, demonstrations through the classical unimodal localization task have generally failed to control for the possible influence of voluntary adjustments based on conscious detection of the intermodal discrepancy. The new paradigm based on a double staircase method that was introduced in the present paper for examining the phenomenon allows the observation of crossmodal bias in the absence of conscious awareness of the discrepancy.

In Experiment 1, it was shown that a central light flashing in synchrony with the target sounds resulted in response reversals occurring at larger eccentricities of the target sounds than was the case in the absence of any such light. Thus, the perceived location of the sounds was shifted in the direction of the flashes. Since the effect was observed at moments when the subject was not aware of the spatial separation between the two inputs, it could not possibly have resulted from voluntary adjustments. On the other hand, the result could not unambiguously be attributed to the synchronization of the flashes with the sounds, because the experimental design confounded synchronization with the more general factor of light interruption. In Experiment 2, we eliminated the possibility of the latter factor as an explanation, by showing that the attraction could be eliminated by desynchronizing the inputs.

Taken together, the two results confirm, with a more straightforward methodology, the suggestion from Bertelson and Radeau's (1981) observation of the occurrence of visual bias of auditory localization in the absence of explicit detection of the intermodal discrepancy. They support the conclusion that the immediate visual bias of auditory location observed previously in less controlled conditions (Bermant \& Welch, 1976; Bertelson et al., 1994; Klemm, 1909; Radeau, 1985, 1992; Radeau \& Bertelson, 1987; Warren et al., 1981) was at least partly due to a phenomenon of automatic attraction between the registered locations of temporally correlated inputs to the two modalities.

A different demonstration of the automatic biasing of apparent auditory location by visual inputs has been provided recently by Driver (1996), who used the traditional "cocktail party" paradigm. The task was to shadow one of two simultaneous word sequences delivered on a single loudspeaker while the face of the talker pronouncing the target sequence was shown on a screen. The critical finding was that performance was considerably better when the screen on which the face appeared was situated at a distance from the loudspeaker than when it was just on top of it. Presumably, the apparent origin of the target auditory items was attracted toward the distant face, thus separating them in space from the distracting items-a condition known to facilitate attentional selection. The distracting items, which were not exactly synchronized with the movements of the face, were probably not attracted, or to a much lesser degree. In this elegant experiment, spatial bias could be inferred indirectly from its effect on recognition. The influence of voluntary adjustments was unlikely because the task involved no reference whatever to location. Although the experiment was not designed specifically to make that point, its results converge nicely with those of the present study, which was based on the usual task of reporting apparent location directly.

\section{REFERENCES}

Bermant, R. I., \& Welch, R. B. (1976). The effect of the degree of visual-auditory stimulus separation upon the spatial interaction of vision and audition. Perceptual \& Motor Skills, 43, 487-493.

BerTelson, P. (1998). Starting from the ventriloquist: The perception of multimodal events. In M. Sabourin, F. I. M. Craik, \& M. Robert (Eds.), Advances in psychological science: Vol. I. Biological and cognitive aspects (pp. 419-439). Hove, U.K.: Psychology Press.

Bertelson, P., \& Aschersleben, G. (1996). Visual bias of auditory location: Evidence for automaticity. Abstracts of the Psychonomic Society, 1, 74

Bertelson, P., \& RadeaU, M. (1976). Ventriloquism, sensory interaction, and response bias: Remarks on the paper by Choe, Welch, Gilford, and Juola. Perception \& Psychophysics, 19, 531-535.

Bertelson, P., \& Radeau, M. (1981). Cross-modal bias and perceptual fusion with auditory-visual spatial discordance. Perception \& Psychophysics, 29, 578-584.

BERTELSON, P., \& RADEAU, M. (1987). Adaptation to auditory-visual conflict: Have top-down influences been overestimated here also? Paper presented at the 2 nd meeting of the European Society for Cognitive Psychology, Madrid.

Bertelson, P., Vroomen, J., Wiegeraad, G., \& de Gelder, B. (1994). Exploring the relation between McGurk interference and ventriloquism. In International Congress on Spoken Language Processing 1994 (pp. 559-562). Yokohama: ICSLP.

CANON, L. K. (1970). Intermodality inconsistency of input and directed attention as determinants of the nature of adaptation. Journal of $E x$ perimental Psychology, 84, 141-147.

Choe, C. S., Welch, R. B., Gilford, R. M., \& Juola, J. F. (1975). The "ventriloquist effect": Visual dominance or response bias? Perception \& Psychophysics, 18, 55-60.

CORNSWEET, T. N. (1962). The staircase-method in psychophysics. American Journal of Psychology, 75, 485-491.

DRIVER, J. (1996). Enhancement of listening by illusory mislocation of speech sounds due to lip-reading. Nature, 381, 66-68.

GraZIano, M. S. A., \& Gross, C. G. (1996). Multiple pathways for processing visual space. In T. Inui \& J. L. McClelland (Eds.) Attention and performance XVI: Information integration in perception and communication (pp. 181-207). Cambridge, MA: MIT Press.

HoWARD, I. P., \& TEMPLETON, W. B. (1966). Human spatial orientation. London: Wiley.

JACK, C. E., \& ThURLOW, W. R. (1973). Effects of degree of visual association and angle of displacement on the "ventriloquism" effect. Perceptual \& Motor Skills, 38, 967-979.

JACKSON, C. V. (1953). Visual factors in auditory localization. Quarterly Journal of Experimental Psychology, 5, 52-65.

KLEMM, O. (1909). Localisation von Sinneneindrücken bei disparaten Nebenreizen. Psychologische Studien, 5, 73-161.

LEVITT, H. (1971). Transformed up-down methods in psychoacoustics. Journal of the Acoustical Society of America, 49, 467-477.

PICK, H. L., JR., WARREN, D. H., \& HAY, J. C. (1969). Sensory conflict in judgments of spatial direction. Perception \& Psychophysics, 6, 203-205.

Predebon, J., \& Woolley, J. S. (1994). The familiar-size cue to depth under reduced-cue viewing conditions. Perception, 23, 1301-1312.

RADEAU, M. (1985). Signal intensity, task context, and auditory-visual interaction. Perception, 14, 571-578.

RADEAU, M. (1992). Cognitive impenetrability in auditory-visual interaction. In J. Alegria, D. Holender, J. Morais, \& M. Radeau (Eds.), Analytic approaches to human cognition (pp. 41-55). Amsterdam: Elsevier.

RADEAU, M., \& BERTELSON, P. (1974). The after-effects of ventriloquism. Quarterly Journal of Experimental Psychology, 26, 63-71.

Radeau, M., \& Bertelson, P. (1976). The effect of a textured visual field on modality dominance in a ventriloquism situation. Perception \& Psychophysics, 20, 227-235.

Radeau, M., \& Bertelson, P. (1977). Adaptation to auditory-visual discordance and ventriloquism in semirealistic situations. Perception \& Psychophysics, 22, 137-146.

RAdeAU, M., \& BerTelson, P. (1987). Auditory-visual interaction and the timing of inputs: Thomas (1941) revisited. Psychological Research, 49, 17-22.

Stein, B. E., \& Meredith, M. A. (1993). The merging of the senses. Cambridge, MA: MIT Press, Bradford Books.

ThOMAS, G. J. (1941). Experimental study of the influence of vision on sound localization. Journal of Experimental Psychology, 28, 167-177.

WARren, D. H., WELCH, R. B., \& MCCARTHY, T. J. (1981). The role of visual-auditory "compellingness" in the ventriloquism effect: Implications for transitivity among the spatial senses. Perception \& Psychophysics, 30, 557-564.

WELCH, R. B. (1978). Perceptual modification: Adapting to altered sensory environments. New York: Academic Press.

WitKin, H. A., WAPNER, S., \& Leventhal, T. (1952). Sound localization with conflicting visual and auditory cues. Journal of Experimental Psychology, 43, 58-67.

\section{NOTES}

1. There is evidence also for the inverse type of relation, auditory bias of visual location. Although it reached significance in at least two experiments (Bertelson \& Radeau, 1981, Experiment 1; Radeau \& Ber- 
telson, 1987), this effect was much smaller, and consequently the majority of studies have, like the present one, focused on the visual bias of audition.

2. The effect considered by Choe et al. (1975) was a translation without deformation along the abscissa of the distributions of subjective sound-light separations for the different types of trials (light left of sound, in same place, or right). It implied that the same subjective distances were affected by synchronization or not, depending on the type of trial on which they occurred. The alternative perceptual hypothesis of a proportional reduction of all subjective distances predicted an effect on $\beta$, as observed.

3. In a more recent experiment (Bertelson \& Radeau, 1987; Radeau, 1992) however, subjects exposed to the same "conceptual conflict" situation showed no bias whatever, while another group working in a "sensory conflict" situation, with synchronized but spatially discordant noise bursts and light flashes, showed the usual visual bias of auditory localization. The semantic effect reported by Pick et al. (1969) is thus less reliable than the sensory one, suggesting an origin of voluntary nature.
4. For a detailed description of the method, see the appendix to the paper (Bertelson \& Radeau, 1981). It is based on the assumption that "same origin" impressions occur on trials on which the perceived intersensory distance falls below a criterion value.

5. The estimation is based on the hypothesis of an ideal observer with an interear distance of $20 \mathrm{~cm}$.

6. In his report on the present manuscript, Dominic Massaro remarked that this fact provides a new argument against the "cue substitution" interpretation of ventriloquism, following which the auditory spatial data are simply replaced by the visual ones (Choe et al., 1975; Howard \& Templeton, 1966, p. 361). If that were the case, response reversals would have occurred under Condition SF from the very first trials of the explorations. Thus, the effect is an integration of visual information with auditory information, not a substitution of auditory by visual information. Other arguments against cue substitution have been developed by Radeau and Bertelson (1976).

(Manuscript received October 7, 1997; revision accepted for publication February 2, 1998.) 